\title{
The ion channel, TRPM2, contributes to the pathogenesis of radiodermatitis
}

\author{
Anne-Laure Perraud ${ }^{1,2}$. Deviyani M. Rao ${ }^{1}$ Elizabeth A. Kosmacek ${ }^{3} \cdot$ Aleksandra Dagunts $^{3}$. \\ Rebecca E. Oberley-Deegan ${ }^{3} \cdot$ Fabienne Gally $^{1}$ (1)
}

Received: 19 January 2018 / Accepted: 20 November 2018 / Published online: 27 November 2018

(c) The Author(s) 2018

\begin{abstract}
Radiodermatitis is a painful side effect for cancer patients undergoing radiotherapy. Irradiation of the skin causes inflammation and breakdown of the epidermis and can lead to significant morbidity and mortality in severe cases, as seen in exposure from accidents or weapons such as "dirty bombs" and ultimately leads to tissue fibrosis. However, the pathogenesis of radiodermatitis is not fully understood. Using a mouse model of radiodermatitis, we showed that the Transient Receptor Potential Melastatin 2 (TRPM2) ion channel plays a significant role in the development of dermatitis following exposure to ionizing radiation. Irradiated TRPM2-deficient mice developed less inflammation, fewer severe skin lesions and decreased fibrosis when compared to wild type mice. The TRPM2-deficient mice also showed a faster recovery period as seen by their increased weight gain post irradiation. Finally, TRPM2-deficient mice exhibited lower systemic inflammation with a reduction in inflammatory cytokines present in the serum. These findings suggest that TRPM2 may be a potential therapeutic target for reducing the severity of radiodermatitis.
\end{abstract}

Keywords Radiodermatitis $\cdot$ Inflammation $\cdot$ Mouse $\cdot$ Transient receptor potential channels (TRP channels) $\cdot$ Trpm2

\section{Introduction}

Radiation therapy is commonly used to treat several types of cancer (Cooperberg et al. 2010; Heminger et al. 2006; Monyak and Levitt 1989; Thomas 1993). However, the major side effect of radiation therapy is skin tissue damage, also known as radiodermatitis, which occurs in $95 \%$ of cancer patients who receive radiation therapy (Salvo et al. 2010). Radiodermatitis can become so severe that cancer treatment is halted until the skin heals which can compromise the effectiveness of treatment. While acute inflammation can be seen within hours of radiation treatment, radiodermatitis takes multiple weeks to develop and its severity progresses

Fabienne Gally

gallyf@njhealth.org

1 Department of Biomedical Research, National Jewish Health, 1400 Jackson St., Room K827, Denver, CO 80206, USA

2 Department of Immunology and Microbiology, University of Colorado Denver, Denver, USA

3 Department of Biochemistry and Molecular Biology, University of Nebraska Medical Center, Omaha, USA over time to erythema, dry or wet desquamation or ulceration. The appearance of these lesions depends on the radiation dose used for treatment as well as biological factors pertaining to the patient, including leukocyte recruitment, release of reactive oxygen species, proteases and other toxic molecules that damage the surrounding tissues. Inflammation is a complex process and contribution to tissue damage and radiodermatitis needs to be better understood.

TRPM2, a regulator of innate immunity and inflammation, is a cationic channel that is activated under conditions of oxidative stress (Knowles et al. 2013; Takahashi et al. 2011). TRPM2 belongs to the family of transient receptor potential (TRP) ion channels. It is referred to as a "chanzyme" because it represents the unique fusion of a $\mathrm{Ca}^{2+}$-permeable pore with an enzymatic region that exhibits residual hydrolase activity toward ADP-ribose (ADPR) (Perraud et al. 2001; Sano et al. 2001). The channel is gated by ADPR (Perraud et al. 2001), which can be produced following NAD depletion in response to radiation-induced oxidative stress. Cells expressing TRPM2 have been found to exhibit an $\mathrm{H}_{2} \mathrm{O}_{2}$-induced $\mathrm{Ca}^{2+}$-influx that was absent in cells lacking the channel (Hara et al. 2002; Perraud et al. 2005). Because TRPM2 is permeable to the universal second 
messenger $\mathrm{Ca}^{2+}$, its expression could result in altered signaling events and inflammatory responses as a result of radiation. Multiple studies have documented the role of TRPM2 in exacerbating cytokine production (Chung et al. 2015; Gally et al. 2018; Ham et al. 2012).

Although radiation-induced skin damage is well known, the mechanisms that cause this reaction are poorly understood. In the present study, we have evaluated the contribution of TRPM2 to radiodermatitis, including irradiated skin damage, lesions and weight loss, and have attributed these responses to increased production of inflammatory mediators.

\section{Materials and methods}

\section{Mice}

Wild-type C57BL/6 and TRPM2-/- (previously described in Knowles et al. 2011) male mice were maintained in our colony and housed under pathogen-free conditions at the National Jewish Health Biological Resource Center. All mice were fed a standard rodent diet and used at 8-12 weeks of age. Experiments were carried out with National Jewish Health Institutional Animal Care and Use Committee (IACUC) approval.

\section{Radiation}

Mice were anesthetized by i.p. injection of Ketamine/Xylazine $(80-10 \mathrm{mg} / \mathrm{kg}$ ), placed in an X-ray irradiator box and shielded with lead so that only the lower pelvic region was exposed to radiation for five consecutive days at a dose of $8 \mathrm{~Gy} /$ day. This dose regimen was chosen because it mimics the radiation therapy regimen of a patient being treated for pelvic cancers (van der Wielen et al. 2009). The mouse weight and skin lesions were documented once a week for 12 weeks.

\section{Clotrimazole and vehicle treatments}

Clotrimazole (CTZ) was purchased from Sigma-Aldrich (cat \# C6019). CTZ was first solubilized in ethanol (50 mg/ml), further diluted in corn oil to reach a $1 \% \mathrm{CTZ}$ containing solution, and filter sterilized using membranes of $0.22 \mu \mathrm{m}$ nominal pore size. Wild-type C57BL/6 male mice were treated topically with this solution. CTZ is clinically very well characterized as it is used as an anti-fungal agent, and its topical application is not toxic. The solution (or ethanol/ corn oil as a vehicle control) was applied twice a week on affected skin areas starting as soon as lesions would appear (4 weeks after irradiation).

\section{Skin evaluation}

The skin lesions were recorded and scored 12 weeks after irradiation for quality as described in (E.J. 2012) (0) normal, (1) erythema, (2) dry desquamation, (3) wet desquamation, and (4) ulceration (image shown in Fig. 1) and length of lesion: (0) $0 \mathrm{~cm}(1)<0.5 \mathrm{~cm},(2)<1 \mathrm{~cm}$, (3) $1-2 \mathrm{~cm}$, and (4) $>2 \mathrm{~cm}$.

\section{Trichrome staining}

Mice were euthanized 12 weeks after irradiation and skin samples were obtained. Tissue specimens were fixed in $10 \%$ formalin and embedded in paraffin. Tissue sections were deparaffinized in xylenes and rehydrated in graded alcohols,
Fig. 1 Criteria for scoring skin tissue lesions following radiation-induced dermatitis. a Representative picture showing the four different effects of radiation on mouse skin (adapted from (Cox et al. 1995)). b The scoring of the tissue lesions was obtained by adding the character of the lesion to the length of the lesion
A

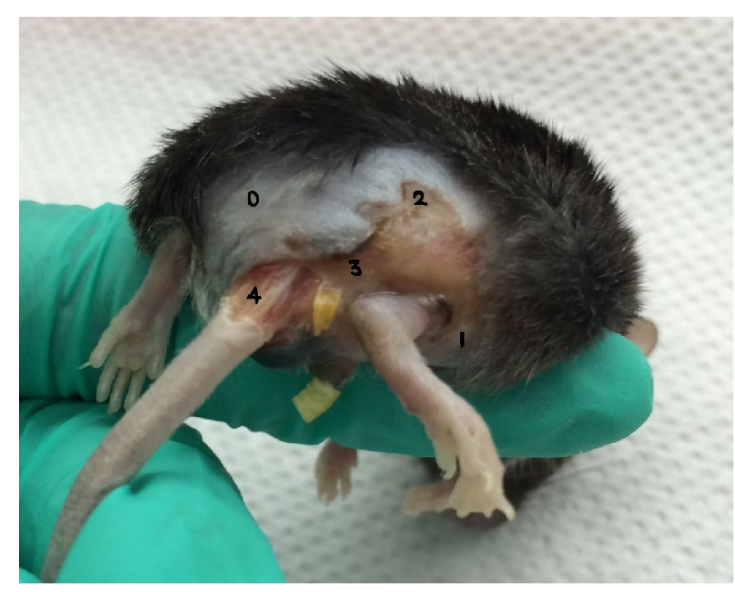

B

\section{Criteria for Scoring Tissue Lesions Following Radiation- Induced Dermatitis}

Character of lesion
no lesion
erythema
dry desquamation
wet desquation
erosion or ulceration
Length of lesion
0 cm
$<0.5 \mathrm{~cm}$

Total tissue lesion score $=$ character of lesion + length of lesion 
then rinsed in running deionized water. Tissues were re-fixed in Bouin's solution for $16 \mathrm{~h}$ at room temperature, and then rinsed for $10 \mathrm{~min}$ in running tap water. Nuclei were stained for $10 \mathrm{~min}$ in Weigert's iron hematoxylin, and thoroughly rinsed in running warm tap water for $10 \mathrm{~min}$. Muscle, cytoplasm and keratin were stained in Biebrich Scarlet-Acid Fuchsin $(0.9-0.1 \%)$ for $5 \mathrm{~min}$ and rinsed before differentiating $15 \mathrm{~min}$ in $2.5 \%$ phosphomolybdic- $2.5 \%$ phosphotungstic acid. Finally, collagen was stained for $8 \mathrm{~min}$ in $2.5 \%$ aniline blue, rinsed, and differentiated for $1 \mathrm{~min}$ in $1 \%$ acetic acid. Sections were dehydrated through graded alcohols, cleared in xylenes, and mounted with Permount (Fisher Scientific, cat. SP15).

\section{Quantification for trichrome staining}

Trichrome stained sections were imaged in brightfield mode, with a $20 \times$ objective, on a Leica DM4000 B LED microscope (Leica Microsystems, Wetzlar, Germany). To measure the collagen density in the skin, each section was imaged over the length of the section requiring ten evenly spaced fields of view. Using ImageJ software, the region of interest (the dermis, excluding hair follicles, sweat glands, blood vessels, and pockets of red blood cells) was selected so that only the area containing collagen was included in the analysis. Next, thresholding was used to select only blue pixels (collagen) and excluded purple/red pixels (immune cells and keratin); white hues were excluded to eliminate holes in the tissue. The collagen density was calculated as the number of pixels representing collagen divided by the total number of pixels in the region of interest (ROI). The percent area of tissue comprised of collagen was averaged for each animal and the mean per group reported.

\section{Quantification for epidermal thickness}

The epidermal layer thickness was quantified using the trichrome staining images. For each image, approximately 20 equally spaced measurements were made along the length of the tissue by drawing a line from the junction of the dermis and epidermis to the edge of the epithelial layer. The pixel value was converted to microns using a factor of 3.84 pixels/ micron. A mean epidermal thickness was calculated for each animal using all images containing epithelium.

\section{Cytokine measurement}

Serum was separated using Z-Gel microtubes (Sarstedt), clotting blood for $30 \mathrm{~min}$ at room temperature, and serum removed by centrifugation (8000 rpm for $5 \mathrm{~min}$ ). Meso Scale Discovery V-Plex Proinflammatory Panel 1 (mouse) plates were used according to the manufacturer's instructions.

\section{Immunostaining}

Tissue sections were deparaffinized in xylenes and rehydrated in graded alcohols, then rinsed in running deionized water. Antigen retrieval was performed by boiling slides in $10 \mathrm{mM}$ sodium citrate buffer, $\mathrm{pH} 6.0$ for $20 \mathrm{~min}$, followed by a 20 -min cool down, and a 10 -min PBS wash. Endogenous peroxidases were quenched for $5 \mathrm{~min}$ in $3 \% \mathrm{H}_{2} \mathrm{O}_{2}$ in PBS, followed by a 5 -min wash. Next, slides were blocked in $10 \%$ goat serum for 30 min followed immediately by $1 \mathrm{~h}$ incubation in primary antibody. Primary antibodies included CD68 (1:100, Abcam, cat. ab125212), CD3 (1:100, Abcam, cat. ab5690), and TRPM2 (1:1000, Abcam, cat. ab11168). Negative stain controls were incubated in blocking buffer without primary antibody for $1 \mathrm{~h}$. Following several washes in PBS, the sections were next incubated in biotinylated goat anti-rabbit secondary antibody (1:200, Vector Labs, cat. BA-1000) then washed in PBS again. Peroxidase activity was associated to the biotinylated secondary antibody using the Vector Labs ABC Kit (cat. PK-4000) by incubation for 30 min in ABC buffer. Finally, DAB substrate was applied to detect the proteins of interest (Vector Labs, cat. SK-4100) for 5-7 min until the brown color was visible under a microscope. Slides were counterstained by briefly dipping in Harris hematoxylin (Sigma-Aldrich, St. Louis, MO, USA, cat. HHS16), then dehydrated through graded alcohols, cleared in xylenes, and mounted with Permount solution.

For both CD3 and CD68 in skin, six random fields of view were captured with a $20 \times$ objective, on a Leica DM4000 B LED microscope. Cells staining a deep brown color were manually counted using the "multi-point" function in ImageJ software. The average cells per field were reported and used for statistical analysis. For TRPM2, serial sections were stained for TRPM2, CD68 and CD3 to determine if TRPM2 expression co-localized in lymphocytes and macrophages.

\section{Statistical analysis}

Data are expressed as mean \pm SEM. One-way analysis of variance was used for multiple comparisons, and Tukey's post hoc test was applied where appropriate. Student's $t$ test was used when only two groups were compared. Differences were considered statistically significant when $p<0.05$.

\section{Results}

Wild-type (WT) and TRPM2 $2^{-1-}$ male mice were irradiated for five sequential days with $8 \mathrm{~Gy} /$ day in the pelvic region. This radiation scheme was used previously in rats (OberleyDeegan et al. 2012) to mimic what a patient with a cancer in the pelvic region would undertake with radiation therapy 
(van der Wielen et al. 2009). At 2 weeks post irradiation, WT mice started to show signs of hair loss and skin erythema in the exposed pelvic region. At 4 weeks, WT mice developed a pronounced loss of hair along with erythema and desquamation of the epidermis while TRPM $2^{-1-}$ mice had just begun to show signs of hair loss. At 12 weeks, while TRPM2 $2^{-1-}$ mice had fully recovered from hair loss, WT mice still exhibited erythema and desquamation (Fig. 2A). Using the scoring system as described in Fig. 1, we determined that TRPM $2^{-1-}$ had significantly less skin lesions than WT mice 12 weeks post irradiation (Fig. 2B).

Weight loss is a well-documented and common side effect from irradiation that can indicate the severity of patient's reaction to radiation therapy which leads us to weigh the mice weekly throughout the 12-week experiment (Fig. 3). While there were no differences in weight loss between WT and TRPM2 $2^{-l-}$ mice up to 5 weeks post-irradiation, TRPM $2^{-/-}$mice recovered and gained weight steadily from 6 to 12 weeks post radiation whereas the WT mice never recovered from their initial weight loss (Fig. 3).

At 12 weeks post irradiation, we performed histological analysis of the skin tissue. Un-irradiated (sham) WT or TRPM $2^{-1-}$ skin did not show any obvious differences (Fig. 4a, left panels). Representative images of lesional irradiated skin regions show that the WT mouse skin appeared more fibrotic and infiltrated with inflammatory cells as compared to TRPM $2^{-/-}$mice (Fig. 4a, right panels). Next, we quantified the fibrosis of the skin by measuring the amount of collagen present using trichrome staining and observed significantly higher levels of collagen in irradiated WT mice as compared to irradiated TRPM $2^{-/-}$mice, while un-irradiated WT or TRPM $2^{-/-}$skin showed no difference (Fig. 4b). We also analyzed alterations in epidermal thickness and measured an increase in irradiated WT mice as compared to irradiated TRPM2 ${ }^{-l-}$ mice, while un-irradiated WT or TRPM2 ${ }^{-/-}$skin showed no difference (Fig. 4c).

As radiation is known to elicit an inflammatory response, we measured the systemic inflammation in the serum of both WT and TRPM $2^{-/-}$mice 4 weeks post irradiation. In accordance with our previous observations, TRPM $2^{-1-}$ mice had reduced inflammatory cytokines, including IL-1 $\beta$, IL-6 and $\mathrm{KC}$ as compared to WT mice (Fig. 5). Additionally,

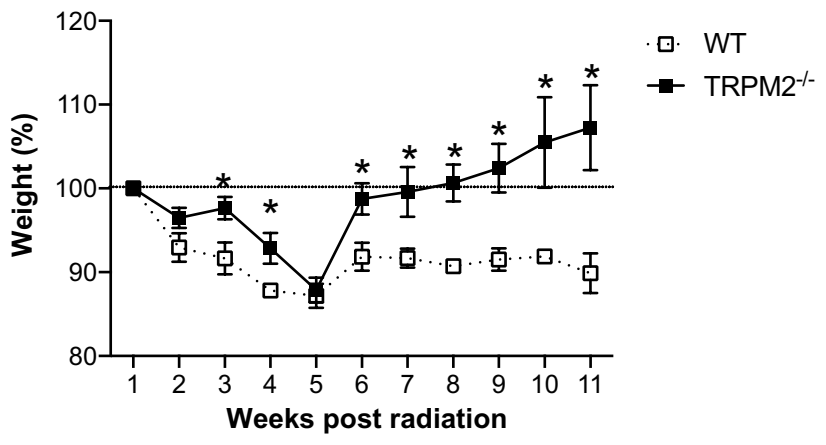

Fig. 3 Weight loss is reduced in TRPM2 $2^{-/-}$mice following irradiation. Weights of irradiated animals throughout the course of the experiment. $N=5-9$ mice per group, ${ }^{*} p<0.05$

we quantified the amount of lymphocytes $(\mathrm{CD} 3+)$ and macrophages $(\mathrm{CD} 68+)$ infiltrating cells in the skin tissue (Figs. 6, 7) 12 weeks post irradiation. While un-irradiated WT or TRPM $2^{-l-}$ skin showed no difference in the amount of lymphocytes or macrophages at baseline, irradiated WT skin showed a significant increase in both, lymphocytes and macrophages, as compared to irradiated TRPM $2^{-1-}$ skin. Taken together, these results suggest that TRPM2-deficiency may play a protective role in radiation-induced damage in part by inhibiting systemic inflammation and leukocyte recruitment.

To further demonstrate that TRPM2 is implicated in radiation-induced inflammation, we stained serial sections of irradiated WT skin tissue for CD3, CD68 and TRPM2 (Fig. 8). Both CD3 positive cells and CD68 positive cells are also positive for TRPM2. These data demonstrate that recruited $\mathrm{T}$ lymphocytes and macrophages following radiation express TRPM2.

To test whether local administration of TRPM2 inhibitors is sufficient to protect against radiodermatitis, we administered a topical TRPM2 inhibitor (clotrimazole) following irradiation of WT mice. As illustrated in Fig. 9a, mice that received clotrimazole lost as much weight as mice that received vehicle treatment. Furthermore, stitched images, using FIJI, of lesional skin showed no difference between vehicle or clotrimazole treatment (Fig. 9b). Since immune
Fig. 2 Radiation-induced dermatitis is reduced in TRPM2 $2^{-/-}$ mice. a Representative photo images of irradiated WT and $\mathrm{TRPM}^{-/-}$mice 12 weeks post irradiation. b Severity of the lesions was quantified using the scoring system described in Fig. 1 on a scale from 0 to 8 . $N=5-9$ mice per group

\section{A}

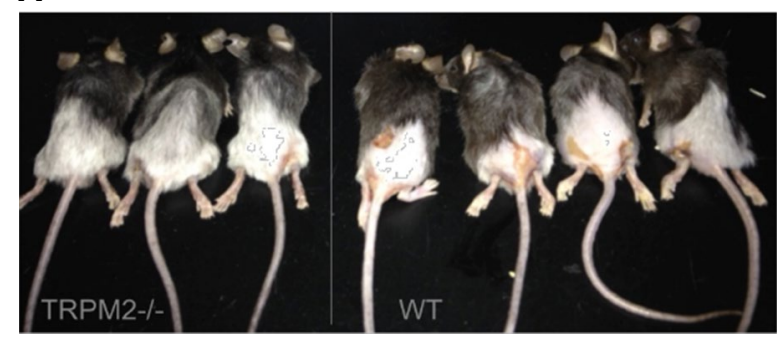

B

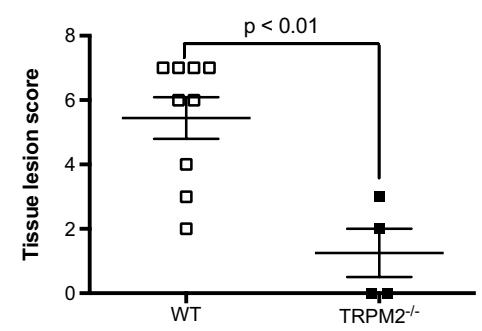


Fig. 4 Radiation-induced skin fibrosis and epidermal thickening is reduced in TRPM $2^{-l-}$ mice. a Representative images of trichrome stained WT and TRPM $^{-/-}$sham and lesional skin 12 weeks post irradiation. Stars indicate sebaceous glands, pounds indicate hair follicles, white arrows indicate inflammatory cells, double arrows indicate the epidermis. Collagen density is proportionate to the intensity of the blue stain. $\mathbf{b}$ Collagen quantification using trichrome staining. c Average epidermal thickness
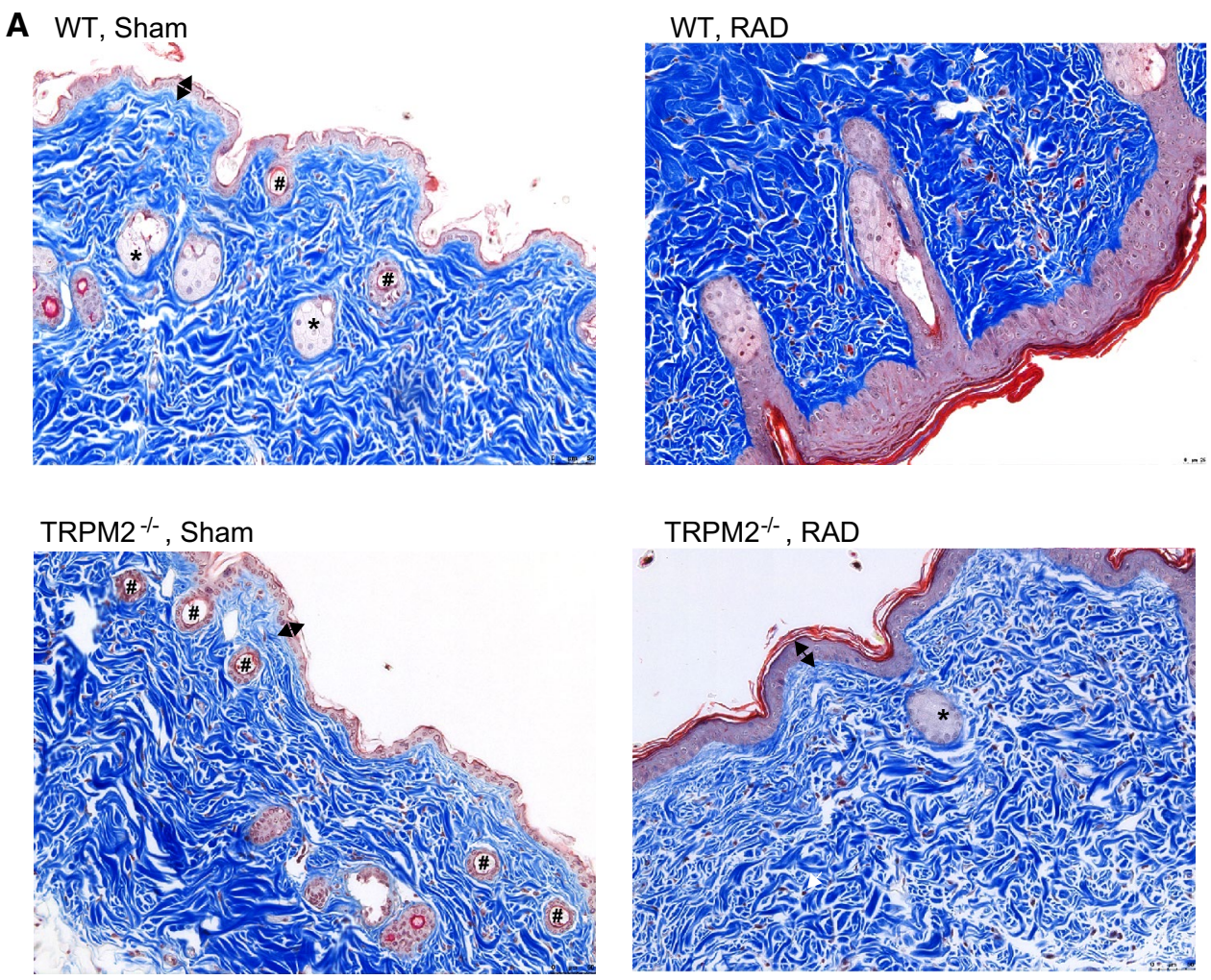

B

Collagen Density

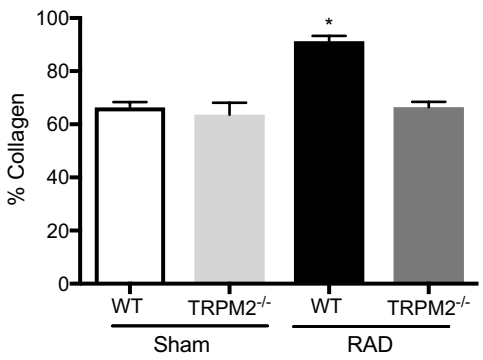

\section{C}

Epidermal thickness

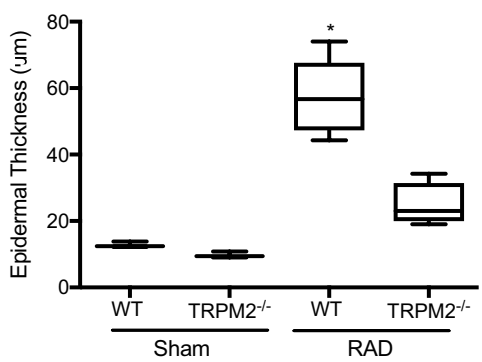

IL-6

A

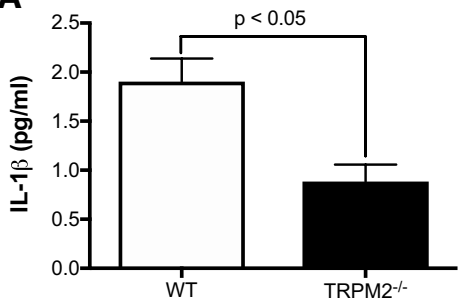

B

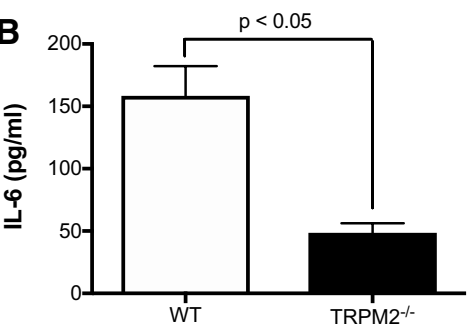

KC

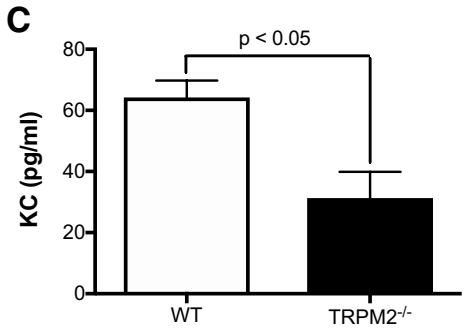

Fig. 5 Inflammatory cytokines are reduced in TRPM2 $2^{-/-}$serum. a IL-1 $\beta$, b IL-6, c KC. $N=5-9$ mice per group

cells require systemic blockade that is not provided by the apical treatment, these data further confirm the implication of TRPM2-induced immune cell recruitment and inflammation. 
Fig. 6 Radiation-induced T cell infiltration is reduced in TRPM $2^{-/-}$mice. a Representative images of CD3 stained WT and TRPM $2^{-1-}$ sham and lesional skin 12 weeks post irradiation. Arrowheads indicate CD3+ cells. b Quantification of CD3 cell numbers per field
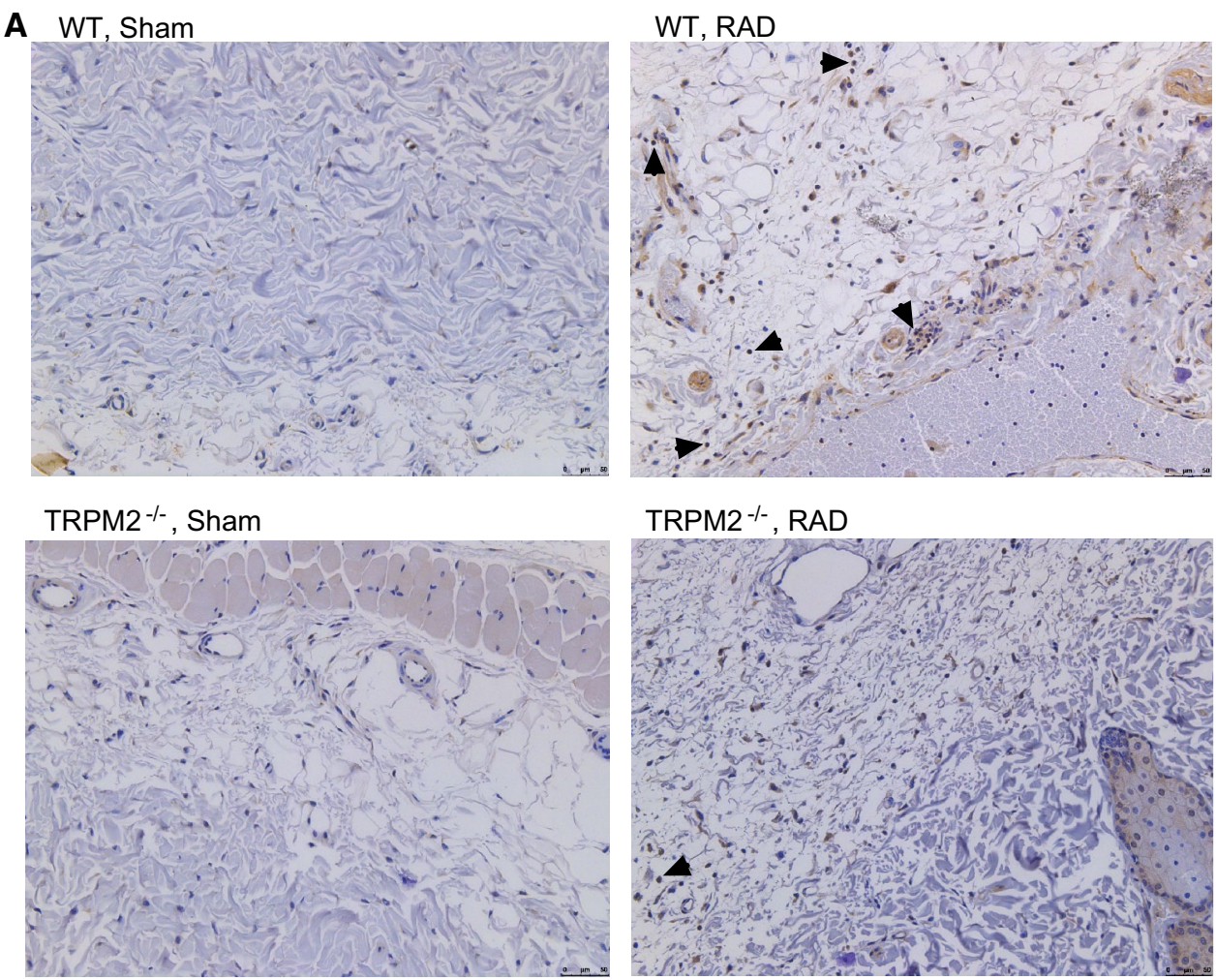

B CD3 cell counts

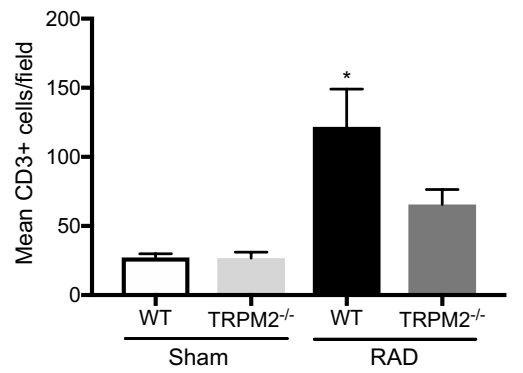

\section{Discussion}

In this study, we have demonstrated that TRPM2-deficiency decreases the severity of various side effects associated with radiation exposure. Specifically, we have shown that TRPM $2^{-/-}$mice are protected from skin damage and overall weight loss associated with lower abdominal radiation exposure. Furthermore, histological analysis of skin lesions showed that TRPM2-deficiency protected the tissue from irradiation-induced damage by limiting the inflammation and the development of fibrosis in irradiated skin. Finally, we showed that TRPM $2^{-/-}$mice had significantly lower circulating inflammatory cytokines and lower leukocyte recruitment, but apical inhibition of TRPM2 had no effect on radiation-induced dermatitis. Taken together, these data suggest that TRPM2 deficiency is protective against radiation-induced skin damage and helps preserve the function of this organ.

The mechanism by which TRPM2-deficiency is most likely protecting the irradiated skin from damage is by decreasing inflammation at the site of exposure. In our studies, radiation-induced TRPM2 ${ }^{-/-}$skin lesions showed less infiltration of inflammatory cells as well as decreased levels of systemic inflammatory cytokines, specifically IL- $1 \beta$, IL-6 and KC. TRPM2 is known to promote inflammation and cytokine production in various situations (Gally et al. 2018; Perraud et al. 2004; Syed Mortadza et al. 2015). Thus, inhibiting TRPM 2 may reduce the severity of radiodermatitis by dampening inflammation systematically and thus halting the vicious cycle of chronic immune activation and tissue injury.

Alternatively, since radiogenic TRPM2 activation and involvement of TRPM2 in DNA damage response has previously been reported (Klumpp et al. 2016; Masumoto 
Fig. 7 Radiation-induced macrophage infiltration is reduced in $\mathrm{TRPM}^{-/-}$mice. a Representative images of CD68 stained WT and TRPM $2^{-/-}$sham and lesional skin 12 weeks post irradiation. Arrowheads indicate CD68+ cells. b Quantification of CD68 cell numbers per field
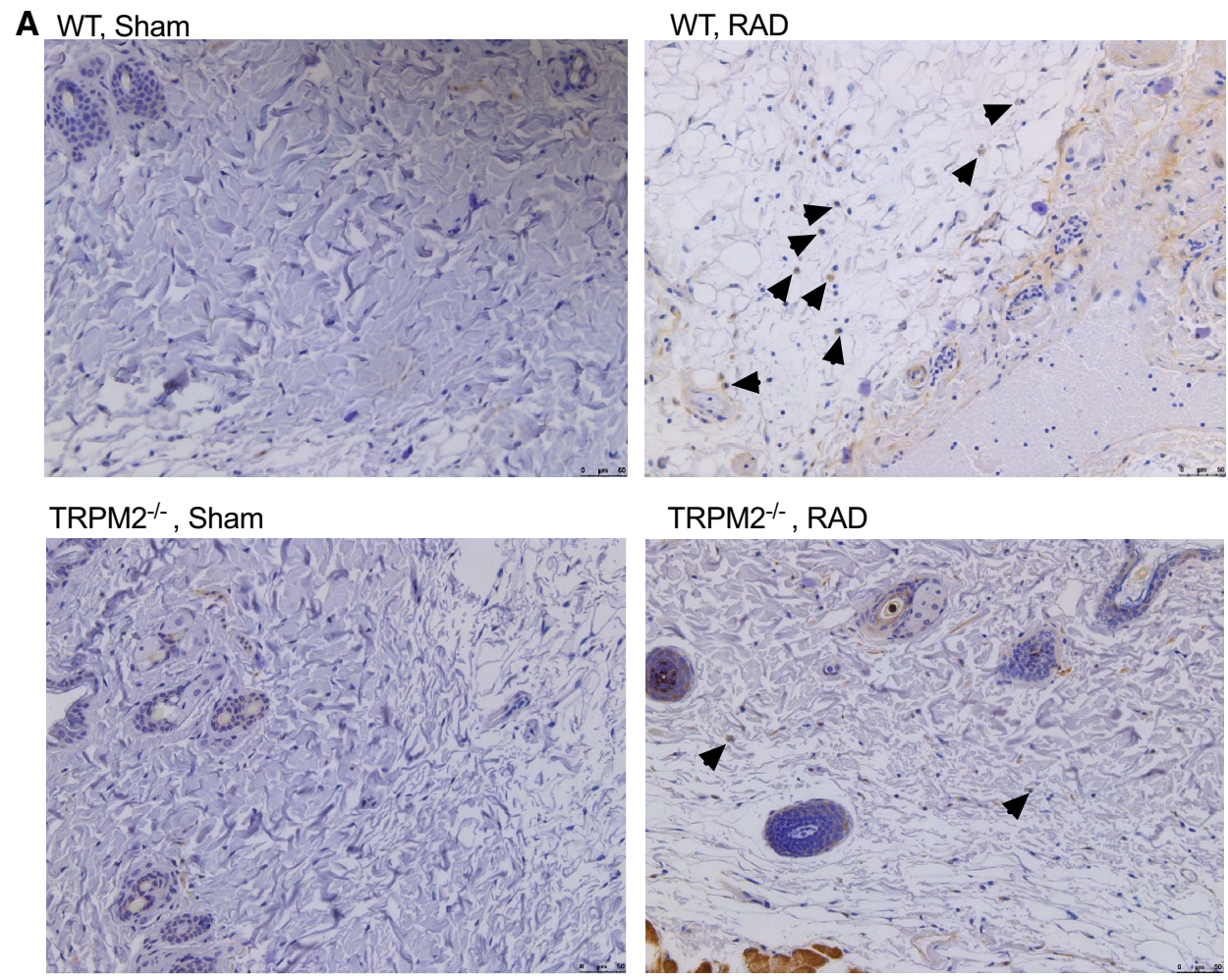

B

CD68 cell counts

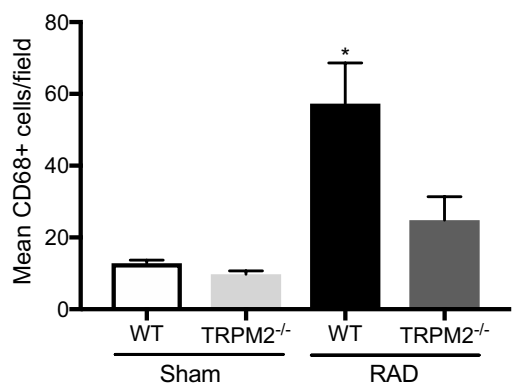

et al. 2013), TRPM2 in the skin might boost immunogenic cell death. While TRPM2 in immune cells would require systemic blockage, local administration of TRPM2 inhibitors would be sufficient to protect against radiation-induced TRPM2 activation and DNA damage. We, thus, administered clotrimazole, a known TRPM2 inhibitor (Hill et al. 2004b), locally to the skin lesions. Clotrimazole did not improve the outcome of radiation-induced dermatitis, thus confirming the importance of TRPM2-induced immune activation.

Ionizing radiation triggers the activation of keratinocytes, fibroblasts and endothelial cells to secrete pro-inflammatory cytokines such as IL-1 $\beta$, IL-6 and KC (Ryan 2012). In turn, IL- $1 \beta$ could activate $\gamma \delta$ T cells and induce IL- 17 expression leading to a pathogenic inflammatory response (Liao et al. 2017). Interestingly, the IL-1 pathway has been shown to play a significant role in the development of radiodermatitis
(Janko et al. 2012). Mice lacking IL-1 or IL-1 receptor have a decrease in inflammation and pathological changes to their skin, similar to what we observed for the TRPM $2^{-/-}$mice (Janko et al. 2012). IL-1 is one of only few cytokines that is induced after skin irradiation and has been implicated in chronic radiodermatitis-induced fibrosis (Liu et al. $2006)$. The reduced IL-1 $\beta$ production that we observed in TRPM $2^{-/-}$mice may therefore be sufficient to protect them from radiodermatitis.

Our findings may have relevance for radiation injury in other tissues since we measured increased levels of inflammatory cytokines in the periphery. TRPM 2 was previously found to contribute to irreversible loss of salivary gland function following irradiation, which is a severe side effect of radiotherapy for head and neck cancers (Liu et al. 2013). In a follow-up study, it was shown that TRPM2 functions as an important regulator of salivary glands, further supporting 
Fig. 8 Infiltrating immune cells express TRPM2. Representative images of irradiated WT skin stained with a CD3, b CD68, c TRPM2, $\mathbf{d}$ no primary TRPM2 antibody (negative control). Circles indicate double positive cells for either CD3 or CD68 and TRPM2 staining
Fig. 9 Apical TRPM2 inhibition did not protect against radiationinduced weight loss and dermatitis. a Weights of WT irradiated animals treated with vehicle or clotrimazole throughout the course of the experiment. $N=5$ mice per group. Representative stitched images of irradiated WT skin treated with $\mathbf{b}$ vehicle or c clotrimazole. Data are representative of two independent experiments
A $\mathrm{CD} 3$

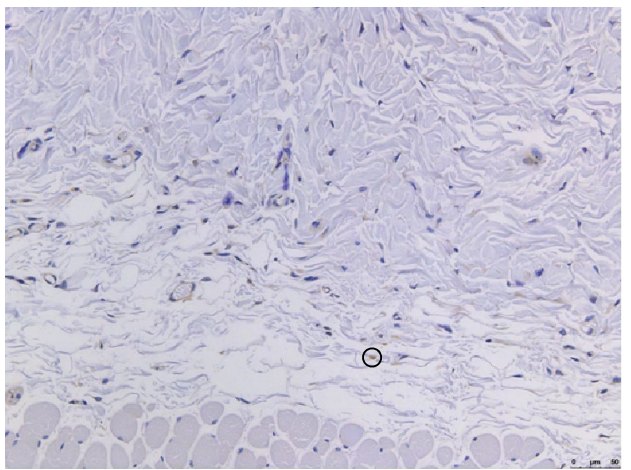

\section{TRPM2}

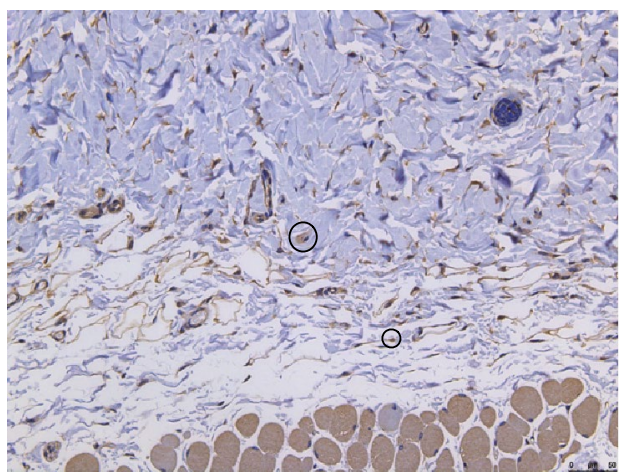

\section{B CD68}

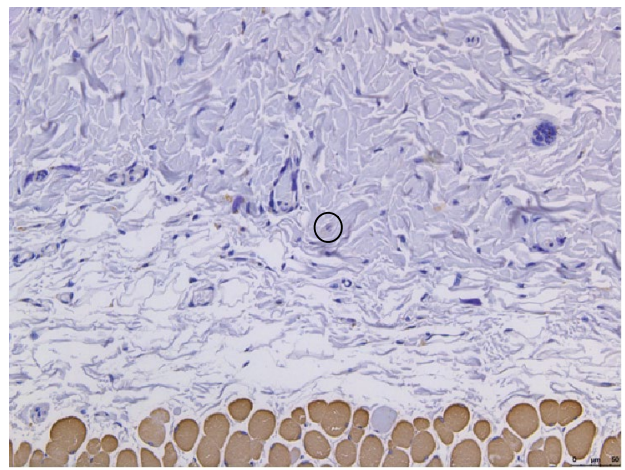

\section{No primary (TRPM2 antibody)}

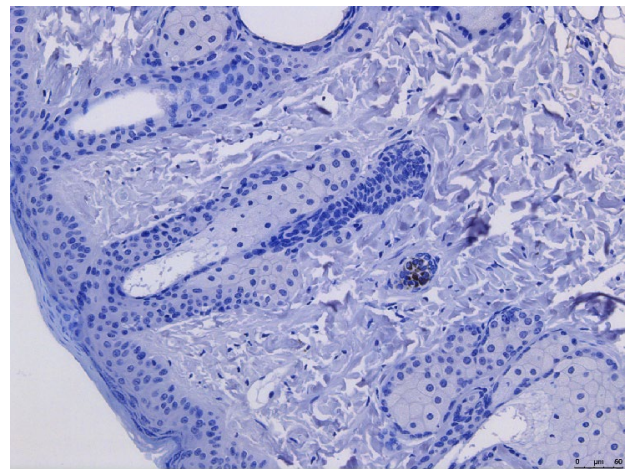

A

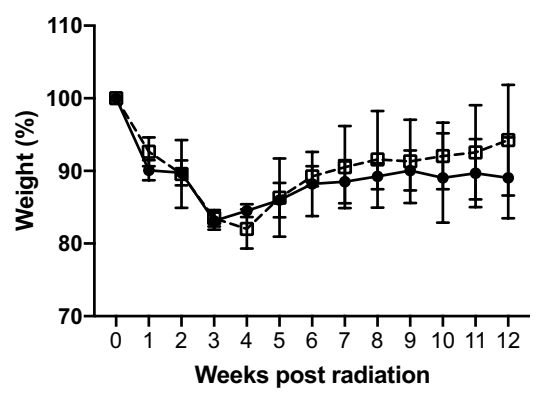

B

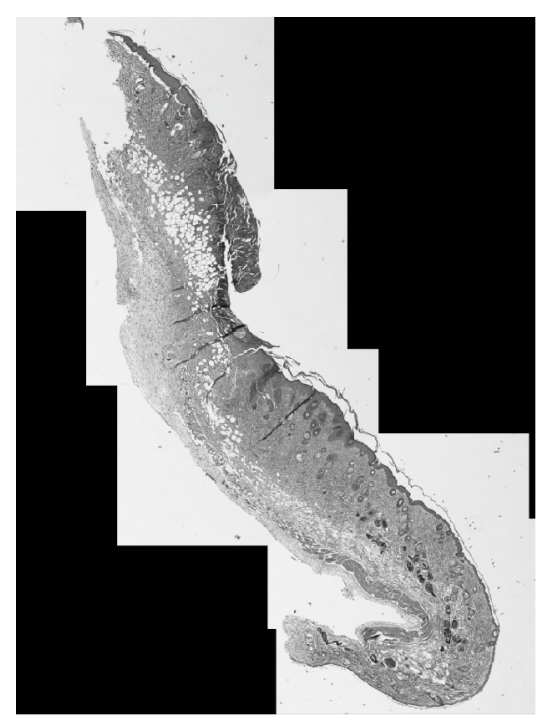

- Vehicle

- - Clotrimazole
C Clotrimazole

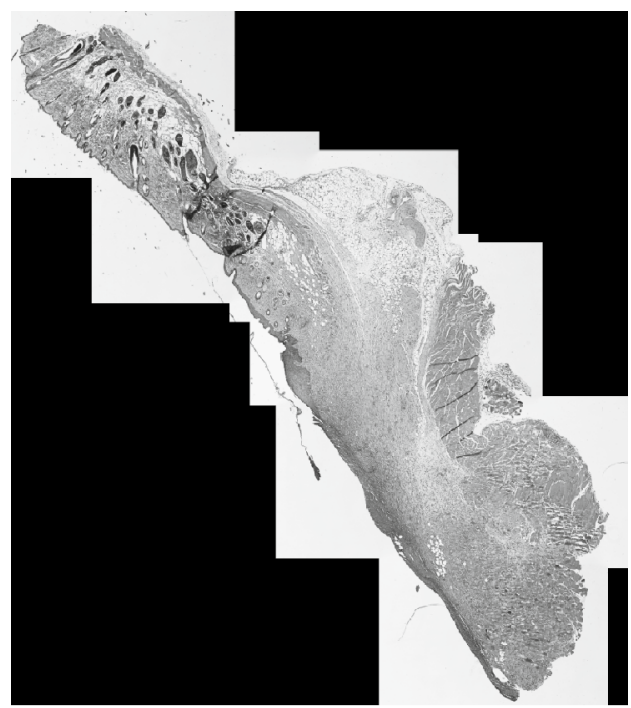


the utility of targeting TRPM2 to protect a wide range of tissues against radiation-mediated injury (Liu et al. 2017).

Several compounds have been shown to inhibit TRPM2 currents. For instance, as stated previously, we used clotrimazole to see if we could prevent radiation-induced skin injury by apically blocking TRPM2. Other compounds such as 2-aminoethoxydiphenyl borate (Togashi et al. 2008) and the anti-fungal econazole (Hill et al. 2004b) have been shown to inhibit ADP-ribose activated TRPM2 currents. Flufenamic acid, a nonsteroidal anti-inflammatory drug, is another TRPM2 inhibitor (Hill et al. 2004a) but it is difficult to dissolve which might be problematic for use at high concentrations. $N$-( $p$-amylcinnamoyl)anthranilic acid inhibits TRPM2 (Kraft et al. 2006), but it also functions as a phospholipase $A_{2}$ inhibitor (Chen et al. 1994). Our studies suggest that a systemic inhibition of TRPM2 would be required to alleviate the effects of radiation on skin damage.

Radiodermatitis is a serious side effect due to radiotherapy to treat many types of tumors found throughout the body, which can lead to the delay of therapeutic treatments. Furthermore, the skin is the first organ that would be affected in a nuclear accident or "dirty bomb" detonation and as such exposed to whole body irradiation. However, given that our understanding of the inflammatory pathways involved in radiodermatitis is still limited, we currently do not have an effective treatment for controlling damage to the skin. Our results emphasize the importance of TRPM2 in mediating radiation-induced inflammatory responses and suggest TRPM2 as a potential target when considering therapeutic interventions for radiodermatitis.

Acknowledgements This work was supported by National Institutes of Health Grants 1R01CA178888, 1R21AI107503-01, and NIH SP20 GM103480 COBRE.

Open Access This article is distributed under the terms of the Creative Commons Attribution 4.0 International License (http://creativeco mmons.org/licenses/by/4.0/), which permits unrestricted use, distribution, and reproduction in any medium, provided you give appropriate credit to the original author(s) and the source, provide a link to the Creative Commons license, and indicate if changes were made.

\section{References}

Chen TH, Lee B, Hsu WH (1994) Arginine vasopressin-stimulated insulin secretion and elevation of intracellular $\mathrm{Ca}++$ concentration in rat insulinoma cells: influences of a phospholipase $\mathrm{C}$ inhibitor 1-[6-[[17 beta-methoxyestra-1,3,5(10)-trien-17-yl]amino] hexyl]-1H-pyrrole-2,5-dione (U-73122) and a phospholipase A2 inhibitor $N$-( $p$-amylcinnamoyl)anthranilic acid. J Pharmacol Exp Ther 270:900-904

Chung MK, Asgar J, Lee J, Shim MS, Dumler C, Ro JY (2015) The role of TRPM2 in hydrogen peroxide-induced expression of inflammatory cytokine and chemokine in rat trigeminal ganglia.
Neuroscience 297:160-169. https://doi.org/10.1016/j.neuroscien ce.2015.03.067

Cooperberg MR, Broering JM, Carroll PR (2010) Time trends and local variation in primary treatment of localized prostate cancer. J Clin Oncol 28:1117-1123. https://doi.org/10.1200/JCO.2009.26.0133

Cox JD, Stetz J, Pajak TF (1995) Toxicity criteria of the Radiation Therapy Oncology Group (RTOG) and the European Organization for Research and Treatment of Cancer (EORTC). Int J Radiat Oncol Biol Phys 31:1341-1346. https://doi.org/10.1016/03603016(95)00060-C

Gally F, Rao DM, Schmitz C, Colvin KL, Yeager ME, Perraud AL (2018) The TRPM2 ion channel contributes to cytokine hyperproduction in a mouse model of down syndrome. Biochim Biophys Acta 1864:126-132. https://doi.org/10.1016/j.bbadis.2017.09.025

Hall EJ (2012) Physics and chemistry of radiation absorption. Radiobiology for the radiologist, 7th edn. Lippincott Williams \& Wilkins, Philadelphia

Ham HY, Hong CW, Lee SN, Kwon MS, Kim YJ, Song DK (2012) Sulfur mustard primes human neutrophils for increased degranulation and stimulates cytokine release via TRPM2/p38 MAPK signaling. Toxicol Appl Pharmacol 258:82-88. https://doi. org/10.1016/j.taap.2011.10.010

Hara Y et al (2002) LTRPC2 Ca2+-permeable channel activated by changes in redox status confers susceptibility to cell death. Mol Cell 9:163-173

Heminger K, Jain V, Kadakia M, Dwarakanath B, Berberich SJ (2006) Altered gene expression induced by ionizing radiation and glycolytic inhibitor 2-deoxy-glucose in a human glioma cell line: implications for radio sensitization. Cancer Biol Ther 5:815-823

Hill K, Benham CD, McNulty S, Randall AD (2004a) Flufenamic acid is a pH-dependent antagonist of TRPM 2 channels. Neuropharmacology 47:450-460. https://doi.org/10.1016/j.neuropharm .2004 .04 .014

Hill K, McNulty S, Randall AD (2004b) Inhibition of TRPM2 channels by the antifungal agents clotrimazole and econazole Naunyn Schmiedebergs. Arch Pharmacol 370:227-237. https://doi. org/10.1007/s00210-004-0981-y

Janko M, Ontiveros F, Fitzgerald TJ, Deng A, DeCicco M, Rock KL (2012) IL-1 generated subsequent to radiation-induced tissue injury contributes to the pathogenesis of radiodermatitis. Radiat Res 178:166-172

Klumpp D, Misovic M, Szteyn K, Shumilina E, Rudner J, Huber SM (2016) Targeting TRPM2 channels impairs radiation-induced cell cycle arrest and fosters cell death of T Cell leukemia cells in a Bcl-2-dependent manner. Oxid Med Cell Longev 2016:8026702. https://doi.org/10.1155/2016/8026702

Knowles $\mathrm{H}$ et al (2011) Transient receptor potential melastatin 2 (TRPM2) ion channel is required for innate immunity against Listeria monocytogenes. Proc Natl Acad Sci USA 108:11578-11583. https://doi.org/10.1073/pnas.1010678108

Knowles H, Li Y, Perraud AL (2013) The TRPM2 ion channel, an oxidative stress and metabolic sensor regulating innate immunity and inflammation. Immunol Res 55:241-248. https://doi.org/10.1007/ s12026-012-8373-8

Kraft R, Grimm C, Frenzel H, Harteneck C (2006) Inhibition of TRPM2 cation channels by $N$-( $p$-amylcinnamoyl)anthranilic acid. Br J Pharmacol 148:264-273. https://doi.org/10.1038/sj.bjp.07067 39

Liao W, Hei TK, Cheng SK (2017) Radiation-induced dermatitis is mediated by IL17-expressing gammadelta T cells. Radiat Res 187:454-464. https://doi.org/10.1667/RR007CC.1

Liu W et al (2006) Interleukin 1beta (IL1B) signaling is a critical component of radiation-induced skin fibrosis. Radiat Res 165:181-191

Liu X et al (2013) Loss of TRPM2 function protects against irradiationinduced salivary gland dysfunction. Nat Commun 4:1515. https:// doi.org/10.1038/ncomms2526 
Liu X et al (2017) Radiation inhibits salivary gland function by promoting STIM1 cleavage by caspase- 3 and loss of SOCE through a TRPM2-dependent pathway. Sci Signal. https://doi.org/10.1126/ scisignal.aal 4064

Masumoto K, Tsukimoto M, Kojima S (2013) Role of TRPM2 and TRPV1 cation channels in cellular responses to radiation-induced DNA damage. Biochim Biophys Acta 1830:3382-3390. https:// doi.org/10.1016/j.bbagen.2013.02.020

Monyak D, Levitt S (1989) The changing role of radiation therapy in the treatment of primary breast cancer. Invest Radiol 24:483-494

Oberley-Deegan RE et al (2012) The antioxidant, MnTE-2-PyP, prevents side-effects incurred by prostate cancer irradiation. PLoS One 7:e44178. https://doi.org/10.1371/journal.pone.0044178

Perraud AL et al (2001) ADP-ribose gating of the calcium-permeable LTRPC 2 channel revealed by Nudix motif homology. Nature 411:595-599

Perraud AL, Knowles HM, Schmitz C (2004) Novel aspects of signaling and ion-homeostasis regulation in immunocytes. The TRPM ion channels and their potential role in modulating the immune response. Mol Immunol 41:657-673. https://doi.org/10.1016/j. molimm.2004.04.013

Perraud AL et al (2005) Accumulation of free ADP-ribose from mitochondria mediates oxidative stress-induced gating of TRPM2 cation channels. J Biol Chem 280:6138-6148

Ryan JL (2012) Ionizing radiation: the good, the bad, and the ugly. J Invest Dermatol 132:985-993. https://doi.org/10.1038/ jid.2011.411
Salvo N et al (2010) Prophylaxis and management of acute radiationinduced skin reactions: a systematic review of the literature. Curr Oncol 17:94-112

Sano Y, Inamura K, Miyake A, Mochizuki S, Yokoi H, Matsushime H, Furuichi K (2001) Immunocyte Ca2+ influx system mediated by LTRPC. Science 293(2):1327-1330

Syed Mortadza SA, Wang L, Li D, Jiang LH (2015) TRPM2 channel-mediated ROS-sensitive $\mathrm{Ca}(2+)$ signaling mechanisms in immune. Cells Front Immunol 6:407. https://doi.org/10.3389/ fimmu.2015.00407

Takahashi N, Kozai D, Kobayashi R, Ebert M, Mori Y (2011) Roles of TRPM2 in oxidative stress. Cell Calcium 50:279-287. https:// doi.org/10.1016/j.ceca.2011.04.006

Thomas GM (1993) Is there a role for consolidation or salvage radiotherapy after chemotherapy in advanced epithelial ovarian cancer? Gynecol Oncol 51:97-103. https://doi.org/10.1006/ gyno.1993.1253

Togashi K, Inada H, Tominaga M (2008) Inhibition of the transient receptor potential cation channel TRPM2 by 2-aminoethoxydiphenyl borate (2-APB). Br J Pharmacol 153:1324-1330. https://doi. org/10.1038/sj.bjp.0707675

van der Wielen GJ et al (2009) Changes in the penile arteries of the rat after fractionated irradiation of the prostate: a pilot study. J Sex Med 6:1908-1913. https://doi.org/10.111 $1 / \mathrm{j} .1743-6109.2009 .01272 . x$ 